\title{
Hedging Entry and Exit Decisions: Activating and Deactivating Barrier Options
}

\author{
LAURENT GAUTHIER ${ }^{\dagger}$ \\ 15 W 73rd St, Apt 2, New York, NY 10023, USA.
}

$\lg @$ ifrance.com

\begin{abstract}
Investment projects and businesses can be entered or exited at a cost, and the theory of real option teaches us how to find optimal activity levels that should trigger entry or exit. However, in practice, different managers or owners operate under different constraints and might apply different thresholds to the same business. We are interested in the hedging of the risk related to the cost of sub-optimal entry or exit. We introduce a new class of derivative products that can hedge this risk. The pricing of these derivatives involves the joint law of a Brownian excursion and its supremum, which is calculated thanks to Bessel processes-related distribution laws.
\end{abstract}

Keywords: Real options, investment decision, Brownian meander, exotic options

\section{Introduction}

Real option theory establishes an analogy between monopolistic investment projects and financial options. An investment project, indeed, contains an option to wait for a better time to invest, depending on the evolution of the random variables that condition the project's profitability (such as market share, commodity prices or labor costs). The classical Net Present Value rule, which prescribes investment as soon as a positive value is generated today, does not take into account the value contained in the option to wait. The option to exit an investment that has proven unattractive also possesses a value. Entry or exit are in most cases costly; there is an often important fixed cost in stopping a manufacturing plant, or restarting it.

Under these conditions, it is well known that the optimal strategy, in terms of the entry or exit from a perpetual investment opportunity, is composed of two levels that will respectively trigger investment and disinvestment, when hit by the relevant variable. Dixit and Pindyck (1994) have presented an extensive survey of real option models, and of the entry/exit decision in particular. The value of an investment, as far as its management follows this rational decision behavior, is a function of the index level (the level of the relevant variables), and depends on whether

$\dagger$ Requests for reprints should be sent to L. Gauthier, 15 W 73rd St, Apt 2, New York, NY 10023, USA. 
the project is activated or not. If the underlying variable upon which the future cashflows of the project depend is traded in the marketplace, the value of the option can be determined thanks to a no arbitrage principle ${ }^{1}$. If it is not traded, one can resort to assuming investors are risk-neutral, and the actual calculation of the project value remains the same, with only a difference in the drift of the underlying variable. This risk-neutral assumption, and how some aversion of risk can be factored into the risk-free rate, is discussed in Trigeorgis (1996).

In practice, the levels at which a firm will enter of exit an investment are not necessarily optimal. McDonald (1999) has shown that there are "rules of thumb" used by corporations managers that allow them to implicitly proxy real options, such as hurdle rates or profitability requirements. This does not mean that many firms are poorly run, but rather that these proxy strategies do a good job of improving the straight Net Present Value rule. One consequence of this observation is that different investors might want to apply different entry and exit levels to the same project, even though they have the same entry costs and assumed underlying dynamics. Different managers may also be able to lower entry or exit costs, or to change the dynamics of the project's stream of cashflows, which would result in different entry and exit levels.

Our goal in this paper is to see how the buyers of a business could "hedge" the difference between their own preferred "optimal" levels and the ones actually in place (whether the difference in the levels comes from a better strategy or from different costs). In our analysis, we introduce a new kind of derivatives, which we call Switch options, and show that they are a welladapted instrument to hedge the risk related to business entry and exit decisions. We will see how Switch options allow the buyer of a business to cancel the risk that, when he implements his new entry/exit strategy (as opposed to the prior strategy followed by the previous owner), he may have to pay immediate entry or exit costs. Since these entry or exit costs depend on whether the firm is active or not when he implements the new strategy, the buyer incurs a random cost. The larger the delay between the buying of the business and when the buyer is actually able to implement his new strategy, the greater the potential discrepancy between the actual activity level of the firm and the optimal activity level.

We define Switch options as path-dependent derivatives written on a single underlying that are activated every time the underlying hits a barrier and deactivated every time it hits another barrier. At maturity, if the option is activated, the holder receives a payoff that is a function of the underlying at that time; if it is not activated, the payoff is a different and lower function of the underlying's price. The number of times such an op- 
tion can be activated and deactivated is not bounded. Unlike a standard barrier option, the Switch option is never totally cancelled when the underlying hits the barrier, as there is always a chance it will go back and hit the other barrier. To our knowledge, such options are not currently traded with significant volume in financial markets.

The second section of the paper focuses on a simple probabilistic approach to calculate the value of an investment with given entry and exit thresholds. In the third section, we give a general pricing formula for Switch options and compare them with standard barrier options. In the fourth section, we analyze the relationship between real options and Switch options, and show how the latter can help hedge the former. The fifth section contains a proof for a central theorem in the third section's approach. This proof makes use of the Brownian Meander. Finally, the sixth section concludes the paper.

\section{Real Options: Entry and Exit Decisions}

The optimal barriers that determine entry or exit decision can be found, and are thoroughly studied in Dixit and Pindyck's book, as well as in the important academic literature on the subject ${ }^{2}$. We propose here a simple probabilistic approach to derive a closed-form solution for the value of an investment with entry and exit. Brennan and Schwartz (1985) proposed a numerical method to calculate the value of an investment project with entry and exit, when the entry and exit levels are determined optimally. Our goal here is to write the value of the investment project as a function of the entry and exit barriers.

If we derive the value of an investment, dependent on the entry and exit levels $a$ and $d$, then, the optimal value of this investment will be its maximum with respect to both $a$ and $d$.

We assume that there is an underlying economic variable $S$, driven by the following process:

$$
\frac{d S_{t}}{S_{t}}=\mu d t+\sigma d B_{t}, S_{0}=x
$$

where $B$ is a Brownian Motion on the measured space $(\Omega, \mathcal{F}, \mathbb{P})$, and $\left(\mathcal{F}_{t}\right)_{t \geq 0}$ is the natural filtration of $B . S$ represents the future stream of cash-flows generated by the investment project, and could therefore be dependent on oil prices (if the project is a refinery), gold prices (in the case of a gold mine), or even on the market share in a given product. As in most of the real-option theory, let us consider that the underlying variable, or one of its derivatives, is traded. 
Since there is only one source of noise, the market is complete. $\mu$ is assumed to be smaller than the risk-free rate $r$ used for discounting the cashflows. Equivalently, we can assume that $\mu=r-\delta$, where $\delta$ is the convenience yield (a continuous dividend paid by the project), and reflects a degree of risk aversion of investors. We suppose there is a running cost of $c$, so that the stream of cashflows generated by the project may sometimes becomes negative. For example, this constant running cost could be the recurrent expenses the firm has to pay to maintain the activity, which are not proportional to profits, such as payroll expenses.

In this case, we derive easily that the value of a perpetual project at time 0 is

$$
\begin{aligned}
\mathbb{E}_{S_{0}}\left[\int_{0}^{\infty} d s e^{-r s}\left(S_{s}-c\right)\right] & =\frac{S_{0}}{\delta}-\frac{c}{r} \\
& =F_{\infty}\left(S_{0}\right)-\frac{c}{r}
\end{aligned}
$$

where $F_{\infty}$ represents the gross revenue from the project.

LEMMA 1 The value $F$ of a perpetual investment project that can be entered at level $a$ and exited at level $d$, for the respective costs of $C_{e}$ and $C_{x}$ verifies:

$$
\begin{aligned}
& F(d)=\frac{\left(\frac{d}{a}\right) \sqrt{\frac{2}{\sigma^{2}}\left(r+\frac{\xi^{2}}{2}\right)}-\frac{\xi}{\sigma}\left(\frac{a}{r-\mu}-\frac{c}{r}-C_{e}\right)}{1-\left(\frac{d}{a}\right)^{2} \sqrt{\frac{2}{\sigma^{2}}\left(r+\frac{\xi^{2}}{2}\right)}} \\
& -\frac{\left(\frac{d}{a}\right)^{2} \sqrt{\frac{2}{\sigma^{2}}\left(r+\frac{\xi^{2}}{2}\right)}\left(\frac{d}{r-\mu}+C_{x}-\frac{c}{r}\right)}{1-\left(\frac{d}{a}\right)^{2} \sqrt{\frac{2}{\sigma^{2}}\left(r+\frac{\xi^{2}}{2}\right)}}
\end{aligned}
$$

and

$$
\begin{gathered}
F(a)=\frac{\frac{a}{r-\mu}-\left(\frac{d}{a}\right)^{\sqrt{\frac{2}{\sigma^{2}}\left(r+\frac{\xi^{2}}{2}\right)}+\frac{\xi}{\sigma}}\left(\frac{d}{r-\mu}+C_{x}-\frac{c}{r}\right)}{1-\left(\frac{d}{a}\right)^{2} \sqrt{\frac{2}{\sigma^{2}}\left(r+\frac{\xi^{2}}{2}\right)}} \\
-\frac{\frac{c}{r}+\left(\frac{d}{a}\right)^{2 \sqrt{\frac{2}{\sigma^{2}}\left(r+\frac{\xi^{2}}{2}\right)}} C_{e}}{1-\left(\frac{d}{a}\right)^{2} \sqrt{\frac{2}{\sigma^{2}}\left(r+\frac{\xi^{2}}{2}\right)}}
\end{gathered}
$$

where $\xi=\frac{1}{\sigma}\left(\mu-\frac{\sigma^{2}}{2}\right)$. 
Proof: We write the value of the investment opportunity just when it has been deactivated as a function of its value just when it has been activated, and reciprocally. Naturally, the value of the investment does not depend on time, only on the level of the underlying variable and whether it is active or not. Consequently, the optimal strategy is time-invariant, meaning that it can be represented by the constant levels $a$ and $d$. Using the strong Markov property of the Brownian Motion, which ensures that Brownian increments before and after a stopping time are independent, gives us:

$$
\begin{aligned}
F(d)= & \mathbb{E}_{d}\left[e^{-r T_{a}(S)}\right]\left(F(a)-C_{e}\right) \\
F(a)= & \mathbb{E}_{a}\left[\int_{0}^{T_{d}(S)} d s e^{-r s}\left(S_{s}-c\right)\right] \\
& +\mathbb{E}_{a}\left[e^{-r T_{d}(S)}\right]\left(F(d)-C_{x}\right) \\
= & \mathbb{E}_{a}\left[\int_{0}^{\infty} d s e^{-r s} S_{s}\right]-\mathbb{E}_{a}\left[\int_{T_{d}(S)}^{\infty} d s e^{-r s} S_{s}\right] \\
& -\frac{c}{r}\left(1-\mathbb{E}_{a}\left[e^{-r T_{d}(S)}\right]\right) \\
& +\mathbb{E}_{a}\left[e^{-r T_{d}(S)}\right]\left(F(d)-C_{x}\right) \\
= & \left(F_{\infty}(a)-\frac{c}{r}\right) \\
& -\mathbb{E}_{a}\left[e^{-r T_{d}(S)}\right]\left(F_{\infty}(d)+C_{x}-\frac{c}{r}\right) \\
& +\mathbb{E}_{a}\left[e^{-r T_{d}(S)}\right] F(d) .
\end{aligned}
$$

By solving for $F(a)$ and $F(d)$ we get

$$
\begin{aligned}
F(d)= & \frac{\mathbb{E}_{d}\left[e^{-r T_{a}(S)}\right]\left(F_{\infty}(a)-\frac{c}{r}\right)}{1-\mathbb{E}_{d}\left[e^{-r T_{a}(S)}\right] \mathbb{E}_{a}\left[e^{-r T_{d}(S)}\right]} \\
& -\frac{\mathbb{E}_{d}\left[e^{-r T_{a}(S)}\right] \mathbb{E}_{a}\left[e^{-r T_{d}(S)}\right] F_{\infty}(d)}{1-\mathbb{E}_{d}\left[e^{-r T_{a}(S)}\right] \mathbb{E}_{a}\left[e^{-r T_{d}(S)}\right]} \\
& -\frac{\mathbb{E}_{d}\left[e^{-r T_{a}(S)}\right] \mathbb{E}_{a}\left[e^{-r T_{d}(S)}\right]\left(C_{x}-\frac{c}{r}\right)}{1-\mathbb{E}_{d}\left[e^{-r T_{a}(S)}\right] \mathbb{E}_{a}\left[e^{-r T_{d}(S)}\right]} \\
& -\frac{\mathbb{E}_{d}\left[e^{-r T_{a}(S)}\right] C_{e}}{1-\mathbb{E}_{d}\left[e^{-r T_{a}(S)}\right] \mathbb{E}_{a}\left[e^{-r T_{d}(S)}\right]}
\end{aligned}
$$


and

$$
\begin{aligned}
F(a)= & \frac{F_{\infty}(a)-\frac{c}{r}-\mathbb{E}_{a}\left[e^{-r T_{d}(S)}\right] F_{\infty}(d)}{1-\mathbb{E}_{d}\left[e^{-r T_{a}(S)}\right] \mathbb{E}_{a}\left[e^{-r T_{d}(S)}\right]} \\
& -\frac{\mathbb{E}_{d}\left[e^{-r T_{a}(S)}\right] \mathbb{E}_{a}\left[e^{-r T_{d}(S)}\right] C_{e}}{1-\mathbb{E}_{d}\left[e^{-r T_{a}(S)}\right] \mathbb{E}_{a}\left[e^{-r T_{d}(S)}\right]} \\
& -\frac{\mathbb{E}_{a}\left[e^{-r T_{d}(S)}\right]\left(C_{x}-\frac{c}{r}\right)}{1-\mathbb{E}_{d}\left[e^{-r T_{a}(S)}\right] \mathbb{E}_{a}\left[e^{-r T_{d}(S)}\right]}
\end{aligned}
$$

We write for $a \geq S_{0}$

$$
\mathbb{E}_{S_{0}}\left[e^{-r T_{a}(S)}\right]=\mathbb{E}_{0}\left[e^{-r T_{\frac{1}{\sigma}} \ln \left(\frac{a}{S_{0}}\right)}\left(B^{\xi}\right)\right]
$$

where $\xi=\frac{1}{\sigma}\left(\mu-\frac{\sigma^{2}}{2}\right)$ and $\left(B_{t}^{\xi}\right)_{t \geq 0}=\left(B_{t}+\xi t\right)_{t \geq 0}$ is the drifted Brownian Motion. We used the fact that

$$
\begin{aligned}
T_{a}(S) & =\inf \left\{t \geq 0, S_{t}=a\right\} \\
& =\inf \left\{t \geq 0, S_{0} \exp \left(\sigma B_{t}^{\xi}\right)=a\right\} \\
& =\inf \left\{t \geq 0, B_{t}^{\xi}=\frac{1}{\sigma} \ln \left(\frac{a}{S_{0}}\right)\right\} .
\end{aligned}
$$

Now, we use the well-known fact that for a Brownian Motion, $\mathbb{E}\left[e^{-\lambda T_{h}}\right]=$ $e^{-|h| \sqrt{2 \lambda}}$, combined with Girsanov's theorem, and we get:

$$
\begin{aligned}
& \mathbb{E}_{S_{0}}\left[e^{-r T_{a}(S)}\right] \\
= & \mathbb{E}_{0}\left[e^{\xi B_{T} \frac{1}{\sigma} \ln \left(\frac{a}{S_{0}}\right)}{ }^{-\frac{\xi^{2}}{2} T \frac{1}{\sigma} \ln \left(\frac{a}{S_{0}}\right)} e^{-r T_{\frac{1}{\sigma} \ln \left(\frac{a}{S_{0}}\right)}(B)}\right] \\
= & \mathbb{E}_{0}\left[e^{\left.\xi \frac{1}{\sigma} \ln \left(\frac{a}{S_{0}}\right) e^{-\left(r+\frac{\xi^{2}}{2}\right) T_{\frac{1}{\sigma} \ln \left(\frac{a}{S_{0}}\right)}(B)}\right]}\right. \\
= & \left(\frac{S_{0}}{a}\right)^{\sqrt{\frac{2}{\sigma^{2}}\left(r+\frac{\xi^{2}}{2}\right)}-\frac{\xi}{\sigma}} .
\end{aligned}
$$

Following the same approach for $d \leq S_{0}$ gets us

$$
\mathbb{E}_{S_{0}}\left[e^{-r T_{d}(S)}\right]=\left(\frac{d}{S_{0}}\right)^{\sqrt{\frac{2}{\sigma^{2}}\left(r+\frac{\xi^{2}}{2}\right)}+\frac{\xi}{\sigma}} .
$$


These calculations allow us to write

$$
\begin{aligned}
F(d)= & \frac{\left(\frac{d}{a}\right)^{\sqrt{\frac{2}{\sigma^{2}}\left(r+\frac{\xi^{2}}{2}\right)}-\frac{\xi}{\sigma}}\left(\frac{a}{r-\mu}-\frac{c}{r}-C_{e}\right)}{1-\left(\frac{d}{a}\right)^{2} \sqrt{\frac{2}{\sigma^{2}}\left(r+\frac{\xi^{2}}{2}\right)}} \\
& -\frac{\left(\frac{d}{a}\right)^{2 \sqrt{\frac{2}{\sigma^{2}}\left(r+\frac{\xi^{2}}{2}\right)}}\left(\frac{d}{r-\mu}+C_{x}-\frac{c}{r}\right)}{1-\left(\frac{d}{a}\right)^{2} \sqrt{\frac{2}{\sigma^{2}}\left(r+\frac{\xi^{2}}{2}\right)}}
\end{aligned}
$$

and

$$
\begin{gathered}
F(a)=\frac{\frac{a}{r-\mu}-\left(\frac{d}{a}\right)^{\sqrt{\frac{2}{\sigma^{2}}\left(r+\frac{\xi^{2}}{2}\right)}+\frac{\xi}{\sigma}}\left(\frac{d}{r-\mu}+C_{x}-\frac{c}{r}\right)}{1-\left(\frac{d}{a}\right)^{2} \sqrt{\frac{2}{\sigma^{2}}\left(r+\frac{\xi^{2}}{2}\right)}} \\
-\frac{\frac{c}{r}+\left(\frac{d}{a}\right)^{2 \sqrt{\frac{2}{\sigma^{2}}\left(r+\frac{\xi^{2}}{2}\right)}} C_{e}}{1-\left(\frac{d}{a}\right)^{2} \sqrt{\frac{2}{\sigma^{2}}\left(r+\frac{\xi^{2}}{2}\right)}}
\end{gathered}
$$

which is the result from the lemma.

We now have the following

Proposition 1 The value of an investment project verifies

$$
\begin{aligned}
F_{\text {deact }}\left(S_{0}\right)= & \left(\frac{S_{0}}{a}\right)^{\sqrt{\frac{2}{\sigma^{2}}\left(r+\frac{\xi^{2}}{2}\right)}-\frac{\xi}{\sigma}}\left(F(a)-C_{e}\right) \\
F_{\text {act }}\left(S_{0}\right)= & \left(\frac{d}{S_{0}}\right)^{\sqrt{\frac{2}{\sigma^{2}}\left(r+\frac{\xi^{2}}{2}\right)}+\frac{\xi}{\sigma}}\left(F(d)-C_{x}-\frac{d}{r-\mu}+\frac{c}{r}\right) \\
& +\frac{S_{0}}{r-\mu}-\frac{c}{r} .
\end{aligned}
$$

Proof: If the project is not activated, then its value is the present value of its value when it is activated, minus the cost of activation:

$$
F_{\text {deact }}\left(S_{0}\right)=\mathbb{E}_{S_{0}}\left[e^{-r T_{a}(S)}\right]\left(F(a)-C_{e}\right) .
$$

If the project is activated, then its value is the present value of the cashflows it will generate until it is stopped, plus its present value deactivated, minus 
the cost of deactivation:

$$
\begin{aligned}
F_{a c t}\left(S_{0}\right)= & \mathbb{E}_{S_{0}}\left[\int_{0}^{T_{d}(S)} d s e^{-r s}\left(S_{s}-c\right)\right] \\
& +\mathbb{E}_{S_{0}}\left[e^{-r T_{d}(S)}\right]\left(F(d)-C_{x}\right) \\
= & \mathbb{E}_{S_{0}}\left[e^{-r T_{d}(S)}\right]\left(F(d)-C_{x}\right) \\
& +F_{\infty}\left(S_{0}\right)-\frac{c}{r} \\
& -\mathbb{E}_{S_{0}}\left[e^{-r T_{d}(S)}\right]\left(F_{\infty}(d)-\frac{c}{r}\right) .
\end{aligned}
$$

We obtain the result by replacing in the above expressions the value for 2 and 3 .

The value of an investment project can be maximized with respect to the entry and exit levels $a$ and $d$. Let us look at an example, where we make the following assumptions:

$$
\begin{aligned}
C_{e} & =500, C_{x}=50, c=93.3 \\
r & =8 \%, \mu=2 \%, \sigma=20 \% \text { and } S_{0}=100 .
\end{aligned}
$$

In these conditions, the value of the project if it is started right away is about 0 (after entry costs). If the project is running and if it is never deactivated, its value is about 500. The following tables show the project's value, depending on the entry levels $a$ and $d$. Note that apart from the case where $a$ or $d$ are very close to $S_{0}$, the project value is not very sensitive to the specific choice of barriers. This would seem to confirm the opinion developed in MacDonald (1999). It appears that as far as the exit level is below 70, the value of the active project is greater by following an entry/exit strategy than by just leaving the project in place (the values in the table are above 500).

Table 1 shows the active value; the different columns represent various inputs for $a$ (above the current index level of 100), while the rows represent various inputs for $d$ (below 100). 
Table 1: Value of an Active Project as a Function of $a$ and $d$

\begin{tabular}{||c|c|c|c|c|c|c|c|c|c||}
\hline$a, d$ & $\mathbf{1 0 0}$ & $\mathbf{1 1 0}$ & $\mathbf{1 2 0}$ & $\mathbf{1 3 0}$ & $\mathbf{1 4 0}$ & $\mathbf{1 5 0}$ & $\mathbf{1 6 0}$ & $\mathbf{1 7 0}$ & $\mathbf{1 8 0}$ \\
\hline $\mathbf{1 0 0}$ & NA & -288 & -84 & 53 & 147 & 212 & 257 & 288 & 309 \\
\hline $\mathbf{9 0}$ & -14 & 141 & 243 & 312 & 360 & 392 & 415 & 430 & 440 \\
\hline $\mathbf{8 0}$ & 305 & 379 & 429 & 463 & 486 & 502 & 513 & 520 & 525 \\
\hline $\mathbf{7 0}$ & 470 & 505 & 529 & 545 & 556 & 564 & 569 & 572 & 574 \\
\hline $\mathbf{6 0}$ & 548 & 564 & 575 & 583 & 588 & 591 & 594 & 596 & 597 \\
\hline $\mathbf{5 0}$ & 576 & 583 & 588 & 591 & 594 & 595 & 596 & 597 & 597 \\
\hline $\mathbf{4 0}$ & 574 & 577 & 579 & 581 & 582 & 582 & 583 & 583 & 583 \\
\hline $\mathbf{3 0}$ & 556 & 557 & 558 & 558 & 559 & 559 & 559 & 559 & 559 \\
\hline $\mathbf{2 0}$ & 532 & 532 & 532 & 532 & 532 & 532 & 532 & 532 & 532 \\
\hline
\end{tabular}

Table 2 shows the value for an inactive project.

Table 2: Value of an Inactive Project as a Function of $a$ and $d$

\begin{tabular}{||c|c|c|c|c|c|c|c|c|c||}
\hline$a, d$ & $\mathbf{1 0 0}$ & $\mathbf{1 1 0}$ & $\mathbf{1 2 0}$ & $\mathbf{1 3 0}$ & $\mathbf{1 4 0}$ & $\mathbf{1 5 0}$ & $\mathbf{1 6 0}$ & $\mathbf{1 7 0}$ & $\mathbf{1 8 0}$ \\
\hline $\mathbf{1 0 0}$ & NA & -520 & -201 & 0 & 131 & 218 & 277 & 317 & 345 \\
\hline $\mathbf{9 0}$ & -638 & -259 & -28 & 119 & 216 & 280 & 324 & 353 & 372 \\
\hline $\mathbf{8 0}$ & -343 & -72 & 96 & 205 & 277 & 325 & 357 & 379 & 392 \\
\hline $\mathbf{7 0}$ & -144 & 53 & 180 & 263 & 318 & 355 & 380 & 396 & 406 \\
\hline $\mathbf{6 0}$ & -23 & 130 & 230 & 297 & 343 & 373 & 393 & 406 & 414 \\
\hline $\mathbf{5 0}$ & 40 & 169 & 255 & 314 & 355 & 382 & 400 & 411 & 420 \\
\hline $\mathbf{4 0}$ & 60 & 180 & 262 & 319 & 357 & 384 & 401 & 412 & 421 \\
\hline $\mathbf{3 0}$ & 52 & 174 & 257 & 315 & 354 & 381 & 399 & 410 & 420 \\
\hline $\mathbf{2 0}$ & 31 & 159 & 247 & 307 & 348 & 377 & 396 & 408 & 418 \\
\hline
\end{tabular}

\section{The Pricing of Switch Options}

Switch options cannot be made out of finite combinations of single or double barrier options. A Switch option cannot either be valued using a two-dimensional partial differential equation, since the value at a barrier depends on whether it has been activated or deactivated, and therefore depends on its value at the preceding barrier hitting time.

The payoff of a Switch option is a function of

- the underlying value at maturity,

- whether or not the option is active at maturity, which depends on whether the underlying value has crossed an activating or deactivating barrier. 
The latter feature can be summarized as: whether the underlying value has crossed a deactivating barrier since it last crossed an activating barrier, or since it was first activated.

So as to price options, the Black-Scholes model standardly assumes, among other things, that it is possible to perfectly replicate the payoff of a derivative product with a self-financing portfolio. If there is only one source of risk, it implies that all the derivatives that can be written on an underlying financial asset are redundant. It makes it possible to hedge any derivative with the underlying. We therefore assume that for the purpose of pricing the Switch options, we are in a "risk-neutral" world, the underlying variable value follows the same process as earlier:

$$
\frac{d S_{t}}{S_{t}}=(r-\delta) d t+\sigma d B_{t}, S_{0}=x .
$$

Let us now define the following path functionals

$$
\begin{aligned}
g_{t}^{a}(X) & =\sup \left\{0 \leq s \leq t: X_{s}=a\right\} \\
m(t, T)(X) & =\inf _{u \in[t, T]} X_{u} \\
T_{a}(X) & =\inf \left\{s \geq 0: X_{s}=a\right\} .
\end{aligned}
$$

Respectively, they are the last time the process crossed level $a$, the minimum, the maximum, and the hitting time of $a$. In the following, we will consider two barriers $a$ and $d$ (activating and deactivating), and we will assume that $a \geq d$, since this is the usual situation in the real option approach. The method to derive the results in the opposite case is the same, and in view of our application to real option theory, it does not serve any purpose

A Switch option can start its life being already activated, or deactivated. A particular case of Switch option would be a special "second chance" knock-out call, that could be reactivated any time after it has been knocked out, just by hitting another level. Such an option would have a zero payoff if it is not activated at maturity.

These options are clearly distinct from classical barrier options

- Even if the "inactivated" payoff is zero, they are never worth zero unless at maturity they are not exercised

- The reactivation feature provides the holder with a sort of insurance against a worst-case scenario.

- In a situation of high volatility, a barrier option would have more chances to be cancelled, whereas if it is also true for the Switch option, it has also more chances of being reactivated. 
We can consider two cases, that is whether the option starts as being active, or inactive. If the option is inactive, then its payoff will be, for a maturity $T$

$$
\begin{aligned}
& p\left(S_{T}\right)\left(\mathbb{I}_{T_{a} \leq T} \mathbb{I}_{m\left(g_{T}^{a}, T\right) \geq d}\right) \\
& +q\left(S_{T}\right)\left(1-\mathbb{I}_{T_{a} \leq T} \mathbb{I}_{m\left(g_{T}^{a}, T\right) \geq d}\right) .
\end{aligned}
$$

This expressions means that if the option starts as inactive, it will pay $p$ if it is activated, and does not hit the deactivating barrier after its latest activation, and $q$ in the other case. The value of an option that starts by being active can be derived from the value of the inactive option. Indeed, the active option will pay: either if it is never deactivated, or if it is deactivated; it will become an option that starts by being inactive at the time it is deactivated.

We can write, thanks to the classical option pricing theory, the price of an inactive option at time 0 and maturity $T$ :

$$
\begin{aligned}
V_{d}(x, T)= & e^{-r T} \mathbb{E}_{x}\left[p\left(S_{T}\right)\left(\mathbb{I}_{T_{a} \leq T} \mathbb{I}_{m\left(g_{T}^{a}, T\right) \geq d}\right)\right] \\
& +e^{-r T} \mathbb{E}_{x}\left[q\left(S_{T}\right)\left(1-\mathbb{I}_{T_{a} \leq T} \mathbb{I}_{m\left(g_{T}^{a}, T\right) \geq d}\right)\right] .
\end{aligned}
$$

We have the following

Proposition 2 The price of the inactive switch option is

$$
\begin{aligned}
V_{d}(x, T)= & \frac{1}{2} e^{-\left(r+\frac{\xi^{2}}{2}\right) T} \int_{0}^{T} \mathbb{P}\left(T_{b} \in d t\right) \int_{0}^{T-t} \mathbb{P}\left(g_{T-t} \in d v\right) \\
& \left\{\int_{0}^{+\infty} d z f(b-\sqrt{T-t-v} z) z e^{-\frac{z^{2}}{2}}\right. \\
& +\int_{0}^{\frac{b-c}{\sqrt{T-t-v}}} d z f(\sqrt{T-t-v} z-b) \\
& \left.\sum_{k \in \mathbb{Z}}\left(z+2 k \frac{b-c}{\sqrt{T-t-v}}\right) e^{-\frac{\left(z+2 k \frac{b-c}{\sqrt{T-t-v}}\right)^{2}}{2}}\right\} \\
+ & e^{-\left(r+\frac{\xi^{2}}{2}\right) T} \int_{-\infty}^{+\infty} \frac{d z e^{-\frac{z^{2}}{2}}}{\sqrt{2 \pi}} q\left(x e^{\sigma z}\right)
\end{aligned}
$$


with

$$
\begin{aligned}
f(y) & =e^{\xi y}\left(p\left(x e^{\sigma y}\right)-q\left(x e^{\sigma y}\right)\right) \\
b & =\frac{1}{\sigma} \ln \left(\frac{a}{x}\right) \\
c & =\frac{1}{\sigma} \ln \left(\frac{d}{x}\right) \\
\xi & =\frac{1}{\sigma}\left(r-\delta-\frac{\sigma^{2}}{2}\right)=\frac{1}{\sigma}\left(\mu-\frac{\sigma^{2}}{2}\right)
\end{aligned}
$$

As in the preceding section, we can chose simplified parameters, so as to make the results clearer. We assume $\mu=\frac{\sigma^{2}}{2}$ so that $\xi=0$. Also, we are interested in a simple payoff function: $p=1$ and $q=0$; then $f=1$. This option pays 1 if it is activated at maturity, and nothing otherwise. Its value in this case is

$$
\begin{aligned}
& \frac{1}{2} e^{-r T} \int_{0}^{T} \mathbb{P}\left(T_{b} \in d t\right) \int_{0}^{T-t} \mathbb{P}\left(g_{T-t} \in d v\right) \\
& \int_{0}^{\frac{b-c}{\sqrt{T-t-v}}} d z \sum_{k \in \mathbb{Z}}\left(z+2 k \frac{b-c}{\sqrt{T-t-v}}\right) e^{-\frac{\left(z+2 k \frac{b-c}{\sqrt{T-t}}\right)^{2}}{2}} \\
= & e^{-r T} \int_{0}^{T} \mathbb{P}\left(T_{b} \in d t\right) \int_{0}^{T-t} \mathbb{P}\left(g_{T-t} \in d v\right) \\
& \sum_{k \in \mathbb{Z}} e^{-2 k^{2} \frac{(b-c)^{2}}{T-t-v}}\left(1-e^{-\frac{1+4 k}{2} \frac{(b-c)^{2}}{T-t-v}}\right) \\
= & e^{-r T} \sum_{k \in \mathbb{Z}} \int_{0}^{T} d t \frac{|b|}{\sqrt{2 \pi t^{3}}} e^{-\frac{b^{2}}{2 t}} \\
& \int_{0}^{T-t} \frac{d v}{\pi \sqrt{v}} e^{-2 k^{2} \frac{(b-c)^{2}}{v}}\left(1-e^{-\frac{1+4 k}{2} \frac{(b-c)^{2}}{v}}\right) .
\end{aligned}
$$

By comparison, the value of a knock-in option that pays 1 if it has been activated (knocked-in) would be:

$$
e^{-r T} \mathbb{P}\left(T_{b} \leq T\right)=e^{-r T} \int_{0}^{T} d t \frac{|b|}{\sqrt{2 \pi t^{3}}} e^{-\frac{b^{2}}{2 t}},
$$

so the difference between the two is

$$
\begin{aligned}
& e^{-r T} \int_{0}^{T} d t \frac{|b|}{\sqrt{2 \pi t^{3}}} e^{-\frac{b^{2}}{2 t}} \\
& \left(1-\sum_{k \in \mathbb{Z}} \int_{0}^{T-t} \frac{d v}{\pi \sqrt{v}} e^{-2 k^{2} \frac{(b-c)^{2}}{v}}\left(1-e^{-\frac{1+4 k}{2} \frac{(b-c)^{2}}{v}}\right)\right) .
\end{aligned}
$$


The proof of the Proposition follows.

Proof: We write $\xi=\frac{1}{\sigma}\left(r-\delta-\frac{\sigma^{2}}{2}\right)$, and we get

$$
\begin{aligned}
V_{d}(x, T)= & e^{-r T} \mathbb{E}\left[\left(p\left(x e^{\sigma B_{T}^{\xi}}\right)-q\left(x e^{\sigma B_{T}^{\xi}}\right)\right)\right. \\
& \left.\mathbb{I}_{T_{\frac{1}{\sigma} \ln \left(\frac{a}{x}\right)} \leq T} \mathbb{I}_{m}\left(g_{T}^{\frac{1}{\sigma} \ln \left(\frac{a}{x}\right)}, T\right) \geq \frac{1}{\sigma} \ln \left(\frac{d}{x}\right)\right] \\
& +e^{-r T} \mathbb{E}\left[q\left(S_{T}\right)\right] .
\end{aligned}
$$

Thanks to Girsanov's theorem, we have

$$
\begin{aligned}
& V_{d}(x, T) \\
& =e^{-r T-\frac{\xi^{2}}{2} T} \mathbb{E}\left[e^{\xi B_{T}}\left(p\left(x e^{\sigma B_{T}}\right)-q\left(x e^{\sigma B_{T}}\right)\right)\right. \\
& \left.\mathbb{I}_{\frac{1}{\sigma} \ln \left(\frac{a}{x}\right)} \leq T \mathbb{I}\left(g_{T}^{\frac{1}{\sigma} \ln \left(\frac{a}{x}\right)}, T\right) \geq \frac{1}{\sigma} \ln \left(\frac{d}{x}\right)\right] \\
& +e^{-r T-\frac{\xi^{2}}{2} T} \mathbb{E}\left[e^{\xi B_{T}} q\left(x e^{\sigma B_{T}}\right)\right] .
\end{aligned}
$$

We will focus on the first term in the above sum. It is natural then to study an expression of the following form, for a Brownian Motion $B$,

$$
\begin{aligned}
& \mathbb{E}\left[f\left(B_{T}\right) \mathbb{I}_{T_{b}(B) \leq T} \mathbb{I}_{m_{g_{T}^{b}, T}(B) \geq c}\right] \\
= & \int_{0}^{T} \mathbb{P}\left(T_{b} \in d t\right) \mathbb{E}\left[f\left(B_{T}\right) \mathbb{I}_{m\left(g_{T}^{b}, T\right) \geq c} \mid T_{b}=t\right] \\
= & \int_{0}^{T} \mathbb{P}\left(T_{b} \in d t\right) \mathbb{E}_{b}\left[f\left(B_{T-t}\right) \mathbb{I}_{m\left(g_{T}^{b}, T\right) \geq c}\right]
\end{aligned}
$$

thanks to the independence of the Brownian paths $\left(B_{t}, t \leq T_{b}\right)$ and $\left(B_{t+T_{b}}-b, t \geq 0\right)$. Now we can write

$$
\begin{aligned}
& \mathbb{E}_{b}\left[f\left(B_{T-t}\right) \mathbb{I}_{\left.m_{g_{T-t}^{b}, T-t} \geq c\right]}\right] \\
= & \mathbb{E}\left[f\left(B_{T-t}+b\right) \mathbb{I}_{m\left(g_{T-t}, T-t\right) \geq c-b}\right] \\
= & \int_{0}^{T-t} \mathbb{P}\left(g_{T-t} \in d v\right) \\
& \mathbb{E}\left[f\left(B_{T-t}+b\right) \mathbb{I}_{m(v, T-t) \geq c-b} \mid g_{T-t}=v\right] .
\end{aligned}
$$


Thus, we have to use the law of the last Brownian excursion away from $b$ before $T-t$. We can write this using the Brownian Meander ${ }^{3}$ and conditioning by whether the excursion straddling $T-t$ is above or under 0 .

$$
\begin{aligned}
& \mathbb{E}\left[f\left(B_{T-t}+b\right) \mathbb{I}_{m(v, T-t) \geq c-b} \mid g_{T-t}=v\right] \\
= & \frac{1}{2} \mathbb{E}\left[f\left(-\sqrt{T-t-v} m_{1}+b\right)\right] \\
& +\frac{1}{2} \mathbb{E}\left[f\left(\sqrt{T-t-v} m_{1}-b\right) \mathbb{I}_{\sup _{u \leq 1} m_{u} \leq \frac{b-c}{\sqrt{T-t-v}}}\right] .
\end{aligned}
$$

Where $m$ is a Meander (do not confuse with the minimum functional that we have noted $m(a, b))$. We have used the scaling property to simplify the expression. Now, we have

$$
\begin{aligned}
& \mathbb{E}\left[f\left(-\sqrt{T-t-v} m_{1}+b\right)\right] \\
= & \int_{0}^{+\infty} d x f(b-\sqrt{T-t-v} x) x e^{-\frac{x^{2}}{2}}
\end{aligned}
$$

using the well-known law (cf Revuz and Yor (1991) or Yor (1995)). Now, using theorem 1 shown in section 5 , we can write

$$
\begin{aligned}
& \mathbb{E}\left[f\left(m_{1}\right) \mathbb{I}_{\left.\sup _{u \leq 1} m_{u} \leq y\right]}\right. \\
= & \int_{0}^{y} d z f(z)(z+2 k y) \sum_{k \in \mathbb{Z}} e^{-\frac{(z+2 k y)^{2}}{2}}
\end{aligned}
$$

which gives, after simplifications:

$$
\begin{aligned}
& \mathbb{E}\left[f\left(B_{T}\right) \mathbb{I}_{T_{b}(B) \leq T} \mathbb{I}_{m\left(g_{T}^{b}, T\right)(B) \geq c}\right] \\
= & \frac{1}{2} \int_{0}^{T} \mathbb{P}\left(T_{b} \in d t\right) \int_{0}^{T-t} \mathbb{P}\left(g_{T-t} \in d v\right) \\
& \left\{\int_{0}^{+\infty} d x f(b-\sqrt{T-t-v} x) x e^{-\frac{x^{2}}{2}}\right. \\
& +\int_{0}^{\frac{b-c}{\sqrt{T-t-v}}} d z f(\sqrt{T-t-v} z-b) \\
& \left.\sum_{k \in \mathbb{Z}}\left(z+2 k \frac{b-c}{\sqrt{T-t-v}}\right) e^{-\frac{\left(z+2 k \frac{b-c}{\sqrt{T-t-v}}\right.}{2}}\right\} .
\end{aligned}
$$


In this expression, the laws of $T_{b}$ and $g_{T-t}$ are known. We have

$$
\begin{aligned}
\mathbb{P}\left(T_{b} \in d t\right) & =d t \frac{|b|}{\sqrt{2 \pi t^{3}}} e^{-\frac{b^{2}}{2 t}} \text { and } \\
\mathbb{P}\left(g_{T-t} \in d v\right) & =\frac{d v}{\pi \sqrt{T-t-v}} .
\end{aligned}
$$

So we can finally write the result and complete the proof.

The price of the active option can also be obtained. As we discussed above, the active option becomes an inactive option when it is deactivated, or just pays at maturity if it is never deactivated. Its price is therefore

$$
\begin{aligned}
V_{a}(x, T)= & \mathbb{E}_{x}\left[e^{-r T_{d}} V_{d}\left(d, T-T_{d}\right) \mathbb{I}_{T_{d} \leq T}\right] \\
& +e^{-r T} \mathbb{E}_{x}\left[p\left(S_{T}\right) \mathbb{I}_{T_{d}>T}\right] .
\end{aligned}
$$

It is very clear in this expression that such a price is decomposed into the price of a classical down and out option $e^{-r T} \mathbb{E}_{x}\left[p\left(S_{T}\right) \mathbb{I}_{T_{d}>T}\right]$ (as it pays only if the cancelling barrier is not hit) and the price of this "second renewable chance" $\mathbb{E}_{x}\left[e^{-r T_{d}} V_{d}\left(d, T-T_{d}\right) \mathbb{I}_{T_{d} \leq T}\right]$, itself matching the value of a Switch option starting deactivated.

Let us write the price of the down and out option as $B^{d o}(x, T)$. We obtain the price of the deactivated Switch option by writing the law of the first hitting time, which is well known. Indeed, we have

$$
\mathbb{P}\left(T_{d} \in d t\right)=\frac{\frac{1}{\sigma} \ln \left(\frac{S_{0}}{d}\right)}{\sqrt{2 \pi t^{3}}} \exp \left(-\frac{1}{2 t}\left(\frac{1}{\sigma} \ln \left(\frac{S_{0}}{d}\right)-\xi t\right)\right)
$$

and it allows us to write

$$
\begin{aligned}
V_{a}(x, T)= & \int_{0}^{T} e^{-r t} \frac{\frac{1}{\sigma} \ln \left(\frac{S_{0}}{d}\right)}{\sqrt{2 \pi t^{3}}} \\
& \exp \left(-\frac{1}{2 t}\left(\frac{1}{\sigma} \ln \left(\frac{S_{0}}{d}\right)-\xi t\right)\right) V_{d}(d, T-t) d t \\
& +B^{d o}(x, T)
\end{aligned}
$$

\section{Relationship Between Switch Options and Real Options.}

\subsection{Switch Options as a Replication Tool}

Let us consider a switch option, the payoff of which at maturity is set to equal the value at that time of an investment project with possible entry and exit. The underlying variable is supposed to be a commodity, traded 
on a market, so that no-arbitrage arguments are valid for pricing purposes. The barriers of the option are chosen so that they are equal to the thresholds of the investment project in question. Therefore, at maturity, whether the investment is active or not, the switch option replicates the project's value. In other words,

$$
\begin{aligned}
& \text { Value of investment project today } \\
& =\text { Value of Switch option today } \\
& \text { whose payoff equals the value } \\
& \text { of the investment project at time } T \text {. }
\end{aligned}
$$

This means that the switch option also replicates the value of the investment at any time since its inception. Hence, buying the switch option is equivalent, in terms of cashflows, to investing and following the optimal entry/exit decision rule. Equivalently, anyone possessing shares in the investment can perfectly hedge them thanks to the switch option. The switch option therefore also constitutes an option to enter into a project, by providing the holder with exactly the necessary amount of cash at maturity to buy the project. It is a financial option written on a real option.

An investment decision typically generates a continuous stream of cashflows, when it is active, and nothing when an exit decision has just been made. If instead the investor buys a switch option, aiming at "exercizing" it at maturity and then buying into the project, he will pay the premium, and then receive nothing. In fact, it is the appreciation in value of the option which compensates for the missing stream of cashflows.

\subsection{Switch Options to Hedge Entry and Exit Costs}

In our analysis, we are more interested in Switch options as a way to hedge the future cost of entering or exiting a business, rather than as replication tools. When a project or a firm changes hands, the new management typically will need to implement new strategies, so that they can extract more value from the business. This could be because the firm was badly run, or because of a special know-how that allows them to reduce current costs. In any case, it is fair to assume that the new management, within the framework of entry/exit decisions and real options, will set up different thresholds from the ones in place.

We believe that in most cases, there is a significant delay between the buying of a business (in fact, that is the instant when the buyer decides to buy the business) and the time when the buyer is able to implement his 
strategy. This delay comes from the time it takes to close the acquisition and restructure the reporting lines in the firm ${ }^{4}$.

Switch options provide a hedging vehicle during this delay. Let us write $a^{\prime}$ and $d^{\prime}$ the activating and deactivating levels following the new strategy, associated with entry and exit costs of $C_{e}^{\prime}$ and $C_{x}^{\prime}$ (which may be different from the costs incurred by the previous owners/managers). Note that if the costs are lower for the buyer, we would expect $a^{\prime} \leq a$ and $d^{\prime} \geq d$. If however the firm was not optimally run, the new optimal barriers could be anywhere with respect to the previous ones.

At time 0, the buyer has decided to acquire the business and possesses a majority of its shares (acquired at the market price, and therefore pricing in the previous strategy). The buyer reckons that he will be able to implement his new strategy only at time $T$. The buyer incurs the risk that, at time $T$, the new optimal strategy will require an immediate change in the firm's activity level:

- if $a^{\prime} \leq a$ then the buyer will need to activate the business and pay $C_{e}^{\prime}$ if $a^{\prime} \leq S_{T} \leq a$ and the business is not active at time $T$

- if $a^{\prime} \geq a$ then the buyer will need to deactivate the business and pay $C_{x}^{\prime}$ if $a^{\prime} \leq S_{T} \leq a$ and the business is active at time $T$

- if $d^{\prime} \geq d$ then the buyer will need to deactivate the business and pay $C_{x}^{\prime}$ if $d^{\prime} \leq S_{T} \leq d$ and the business is active at time $T$

- $\mathrm{f} d^{\prime} \leq d$ then the buyer will need to activate the business and pay $C_{e}^{\prime}$ if $d^{\prime} \leq S_{T} \leq d$ and the business is not active at time $T$

A simple Switch option allows us to hedge these risks. For example, if $a^{\prime} \geq a$ and $d^{\prime} \leq d$, then the buyer would need to be long one Switch option that pays

- $C_{e}^{\prime}$ if deactivated with levels $a$ and $d$ and if $d^{\prime} \leq S_{T} \leq d$

- $C_{x}^{\prime}$ if activated with levels $a$ and $d$ and if $a^{\prime} \leq S_{T} \leq a$.

The value of the option at time zero would therefore be given by Proposition 2 , with

$$
\begin{aligned}
& p(z)=C_{x}^{\prime} \mathbb{I}_{a^{\prime} \geq z \geq a} \\
& q(z)=C_{e}^{\prime} I_{d^{\prime} \geq z \geq d}
\end{aligned}
$$

To illustrate this calculation, let us price one leg of this Switch option. We are interested in the part of the option that pays $C_{e}^{\prime}$ if the project is 
not activated at time $T$ and $d^{\prime} \leq S_{T} \leq d$. Let us see what happens if the new owner wants to lower the exit threshold significantly. This would make sense if the business was previously run with an excessively high exit level: in this case, exiting often costs a lot, based on the example numbers we showed in the first section. Let us assume that $\mu=\frac{\sigma^{2}}{2}$ so as to simplify the calculations.

If we use the same parameters as in the numerical examples of the first section, with $a=120$ and $d=80$, and $d^{\prime}=40$ (so the inactive project has a value of about 96 today), we find that the option is worth about $15 \%$ of $C_{e}^{\prime}$. Therefore, if $C_{e}^{\prime}$ is the same as $C_{x}$ (say 500), then the new owner could pay 75 for the Switch option. This option will pay the new owner the entry cost of 500 in a year, if and only if the business is not active while it should be active. Since the value of the project with the new barriers of 120 and 40 is 262 , that is 166 over its current cost with the sub-optimal barriers of 120 and 80, the cost of buying the Switch option is well compensated for.

\section{The Joint Law of the Brownian Meander and Its Running Supremum}

We have the following:

THEOREM 1 For any measurable positive or bounded function $f$ we have

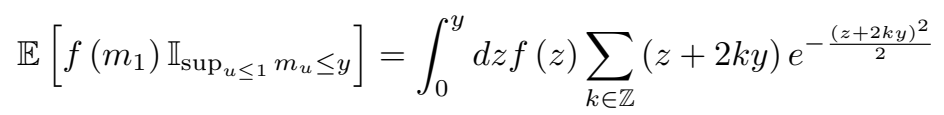

where $m$ is a Brownian meander $\left(m_{u}=\frac{1}{\sqrt{t-g_{t}}}\left|B_{g_{t}+u\left(t-g_{t}\right)}\right|, 0 \leq u \leq 1\right)$ for any positive $t$.

As could be expected, we can check that

$$
\begin{aligned}
\lim _{y \rightarrow \infty} \mathbb{E}\left[f\left(m_{1}\right) \mathbb{I}_{\sup _{u \leq 1} m_{u} \leq y}\right] & =\int_{0}^{\infty} d z f(z) z e^{-\frac{z^{2}}{2}}=\mathbb{E}\left[f\left(m_{1}\right)\right] \text { and } \\
\lim _{y \rightarrow 0} \mathbb{E}\left[f\left(m_{1}\right) \mathbb{I}_{\sup _{u \leq 1} m_{u} \leq y}\right] & =0 .
\end{aligned}
$$

Proof: The proof relies on Imhof's theorem and on the explicit expression of running supremum densities for Bessel-3 processes. These densities are known and can be directly obtained from Borodin and Salminen(1996). They give the joint law of a Bessel-3 process starting from $x>0$ and its 
running maximum (formula 1.1.8, p. 317):

$$
\begin{aligned}
& \mathbb{P}_{x}\left(R_{t} \in d z, \sup _{s \leq t} R_{s} \leq y\right) \\
= & \frac{z}{x \sqrt{2 \pi t}} \sum_{k \in \mathbb{Z}}\left(e^{-\frac{(z-x+2 k y)^{2}}{2 t}}-e^{-\frac{(z+x+2 k y)^{2}}{2 t}}\right) d z .
\end{aligned}
$$

A limit calculation gives the law for a Bessel-3 process starting from 0 .

$$
\mathbb{P}_{0}\left(R_{1} \in d z, \sup _{s \leq 1} R_{s} \leq y\right)=\mathbb{I}_{z \leq y} \frac{z \sqrt{2}}{\sqrt{\pi}} \sum_{k \in \mathbb{Z}}(z+2 k y) e^{-\frac{(z+2 k y)^{2}}{2}} d z .
$$

Thanks to Imhof's theorem (see Yor (1997) or Imhof (1984)), we have a relationship between the Brownian meander and a Bessel-3 process. Namely, we have

$$
\left.M\right|_{\mathcal{F}_{1}}=\left.\left(\sqrt{\frac{\pi}{2}} \frac{1}{X_{1}}\right) R_{0}^{(3)}\right|_{\mathcal{F}_{1}}
$$

where $M$ is the law of the meander between 0 and 1 and $R$ is the law of a Bessel-3 process starting from zero up to 1 . Applying this result gives

$$
\begin{aligned}
& \mathbb{E}\left[f\left(m_{1}\right) \mathbb{I}_{\left.\sup _{u \leq 1} m_{u} \leq y\right]}\right] \\
= & \mathbb{E}_{0}\left[f\left(R_{1}\right) \sqrt{\frac{\pi}{2}} \frac{1}{R_{1}} \mathbb{I}_{\sup _{u \leq 1} R_{u} \leq y}\right] \\
= & \int_{0}^{y} d z f(z) \sum_{k \in \mathbb{Z}}(z+2 k y) e^{-\frac{(z+2 k y)^{2}}{2}} \\
& \text { which can also be written } \\
= & \sqrt{2 \pi} \sum_{k \in \mathbb{Z}} \mathbb{E}\left[f(N-2 k y) N \mathbb{I}_{(2 k+1) y \geq N \geq 2 k y}\right] \\
& \text { for } N \text { a normal Gaussian. }
\end{aligned}
$$

This ends the proof.

\section{Concluding Remarks}

We have proposed a new class of barrier derivatives, Switch options, that allow to mitigate the losses due to the "knock-out" effect of classical barrier options. These derivative products also constitute a hedging tool of the business risk linked to entry or exit decisions. Switch options can replicate the exit or entry costs that a buyer of a business might have to pay so as to implement his optimal entry and exit strategy. 
We required that the underlying business variable be traded, which would restraint the use of these derivatives mostly to commodities firms, unless investors are assumed to be risk-neutral. As a tool to price Switch options, we have derived the joint law of the Brownian Meander and its running maximum.

\section{Notes}

1. As in the case of financial options, any future stream of cashflows that can be replicated can be priced, and its value equals the expectation of these cashflows under the risk-neutral probability. See Dixit and Pindyck (1994) or Trigeorgis (1996) for an exposition of these principles applied to real options.

2. Dixit and Pindyck's book contains an extensive review of the literature.

3. For a precise definition and some comments, refer to the book by Revuz and Yor (1991), Chapter XII, p. 454, Exercise 3.8

4. Recent examples, such as the buying of JP Morgan by Chase, or DLJ by CSFB, illutrate the delay can easily be of 6 months.

\section{References}

1. A. Borodin and P. Salminen. Handbook of Brownian Motion - Facts and Formulae. Birkhauser. Basel Boston Berlin. 1996.

2. M. J. Brennan and E. S. Schwartz. Evaluating Natural Resources Investments. Journal of Business, 58(2), 135-157. 1985.

3. A. Dixit and R. Pindyck. Investment under Uncertainty. Princeton University Press. Princeton. 1994.

4. J.-P. Imhof. Density Factorizations for Brownian Motion, Meander, and the ThreeDimensional Bessel Process, and Applications. Journal of Applied Probability, 21, 500-510. 1984

5. R. L. McDonald. Real Options and Rules of Thumb in Capital Budgeting, in Innovation, Infrastructure, and Strategic Options. Eds. M. J. Brennan and L. Trigeorgis. Oxford University Press. London. 1999.

6. D. Revuz and M. Yor. Continuous Martingales and Brownian Motion. SpringerVerlag. Berlin Heidelberg New York. 1991.

7. L. Trigerogis. Real Options, Managerial Flexibility and Strategy in Resource Allocation. MIT Press. Cambridge. 1996

8. M. Yor. Local Times and Excursion for Brownian Motion: a Concise Introduction. Lecciones in Matematicas, Universidad Central de Venezuela. 1997. 


\section{Mathematical Problems in Engineering}

\section{Special Issue on}

\section{Time-Dependent Billiards}

\section{Call for Papers}

This subject has been extensively studied in the past years for one-, two-, and three-dimensional space. Additionally, such dynamical systems can exhibit a very important and still unexplained phenomenon, called as the Fermi acceleration phenomenon. Basically, the phenomenon of Fermi acceleration (FA) is a process in which a classical particle can acquire unbounded energy from collisions with a heavy moving wall. This phenomenon was originally proposed by Enrico Fermi in 1949 as a possible explanation of the origin of the large energies of the cosmic particles. His original model was then modified and considered under different approaches and using many versions. Moreover, applications of FA have been of a large broad interest in many different fields of science including plasma physics, astrophysics, atomic physics, optics, and time-dependent billiard problems and they are useful for controlling chaos in Engineering and dynamical systems exhibiting chaos (both conservative and dissipative chaos).

We intend to publish in this special issue papers reporting research on time-dependent billiards. The topic includes both conservative and dissipative dynamics. Papers discussing dynamical properties, statistical and mathematical results, stability investigation of the phase space structure, the phenomenon of Fermi acceleration, conditions for having suppression of Fermi acceleration, and computational and numerical methods for exploring these structures and applications are welcome.

To be acceptable for publication in the special issue of Mathematical Problems in Engineering, papers must make significant, original, and correct contributions to one or more of the topics above mentioned. Mathematical papers regarding the topics above are also welcome.

Authors should follow the Mathematical Problems in Engineering manuscript format described at http://www .hindawi.com/journals/mpe/. Prospective authors should submit an electronic copy of their complete manuscript through the journal Manuscript Tracking System at http:// mts.hindawi.com/ according to the following timetable:

\begin{tabular}{|l|l|}
\hline Manuscript Due & December 1,2008 \\
\hline First Round of Reviews & March 1, 2009 \\
\hline Publication Date & June 1, 2009 \\
\hline
\end{tabular}

\section{Guest Editors}

Edson Denis Leonel, Departamento de Estatística, Matemática Aplicada e Computação, Instituto de Geociências e Ciências Exatas, Universidade Estadual Paulista, Avenida 24A, 1515 Bela Vista, 13506-700 Rio Claro, SP, Brazil ; edleonel@rc.unesp.br

Alexander Loskutov, Physics Faculty, Moscow State University, Vorob'evy Gory, Moscow 119992, Russia; loskutov@chaos.phys.msu.ru 